\title{
MULTI-PASS SAR INTERFEROMETRY FOR 3D RECONSTRUCTION OF COMPLEX MOUNTAINOUS AREAS BASED ON ROBUST LOW RANK TENSOR DECOMPOSITION
}

\author{
Jian Kang ${ }^{1}$, Yuanyuan Wang ${ }^{1}$, Xiao Xiang Zhu ${ }^{1,2}$ \\ (1) Signal Processing in Earth Observation, Technical University of Munich \\ (2) Remote Sensing Technology Institute, Germany Aerospace Center (DLR)
}

\begin{abstract}
During the past decades, multi-pass SAR interferometry (InSAR) techniques have been developed for retrieving geophysical parameters such as elevation, over large areas. Conventional method such as periodogram usually requires a fairly large SAR image stack (usually in the order of tens), in order to achieve reliable estimates of these parameters. However, when it comes to large-area processing, it is time-consuming and luxury to obtain a sufficient number of SAR images for the reconstruction. In this paper, we demonstrate a novel multi-pass InSAR method for 3D reconstruction using low rank tensor decomposition. By exploiting the low rank prior knowledge in the multi-pass InSAR stack, simulations show that the proposed method can improve the accuracy of elevation estimates by a factor of two, compared to the stateof-the-art InSAR filtering methods, such as SqueeSAR. The capability of the proposed algorithm is also demonstrated on real data using one TanDEM-X InSAR stack of a complex mountainous area.
\end{abstract}

Index Terms - Low Rank, Multi-pass InSAR, DEM, TanDEM-X

\section{INTRODUCTION}

Multi-pass InSAR is a class of established techniques for retrieving large-area surface displacement and Digital Elevation Model (DEM). In order to achieve reliable estimates of those geophysical parameters, conventional methods, such as PSInSAR [1], usually require a fairly large SAR image stack (usually in the order of tens). However, when it comes to large-area processing, such as global DEM generation [2], it is computationally expensive and cost inefficient to acquire and process stacks of large number of SAR images for the reconstruction.

In this paper, we propose a novel elevation estimation method for complex mountainous areas with application to TanDEM-X data. By exploiting the inherent low rank prior knowledge of complex InSAR stacks [3], the proposed method can achieve a reliable estimation accuracy with a limited number of SAR images (less than ten).
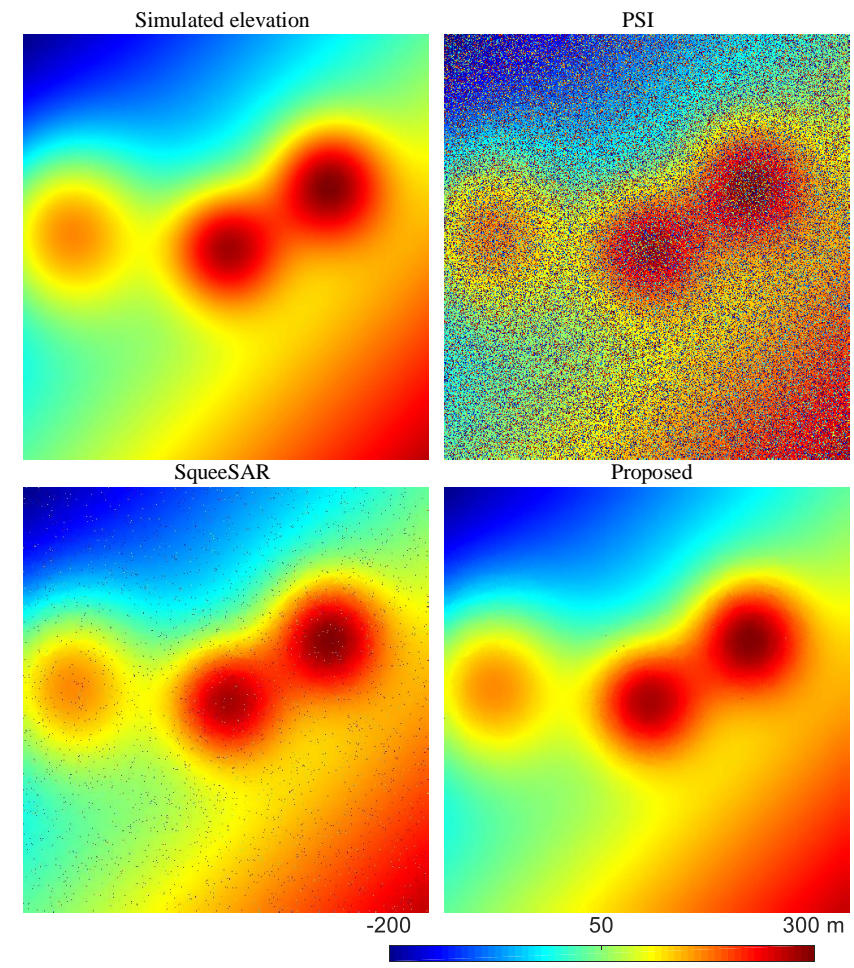

Fig. 1. Simulated ground truth elevation map and the estimated results by PSI, SqueeSAR and the proposed method.

To this end, the proposed method for 3D reconstruction of complex mountainous areas contains the following steps:

- Statistically homogeneous pixels selection based on Anderson-Darling test [4].

- Phase history retrieval of Distributed Scatterers (DS) by the phase triangulation algorithm [5].

- Apply robust low rank tensor decomposition to the filtered InSAR stack.

- Reconstruct elevations based on the outlier-free InSAR stack via periodogram. 


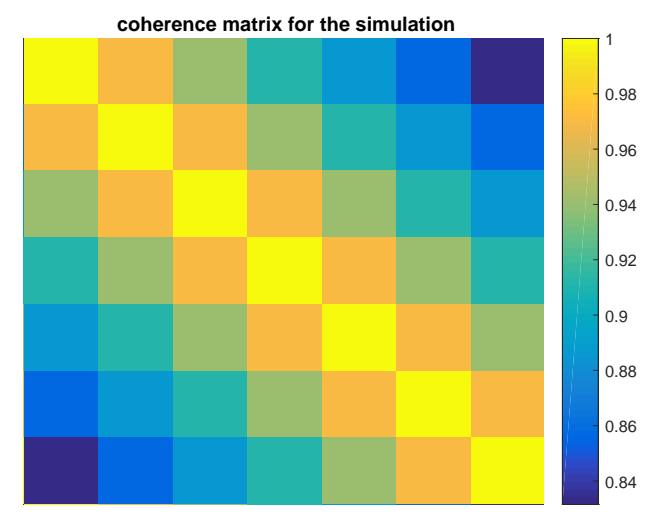

Fig. 2. Coherence matrix for DS simulation

\section{TENSOR MODEL OF MULTI-PASS INSAR STACK}

As proposed in our previous work [3, 6, 7], an InSAR data stack can be represented by a 3-mode tensor: $\mathcal{G} \in \mathbb{C}^{I_{1} \times I_{2} \times I_{3}}$, where $I_{1}$ and $I_{2}$ represent the spatial dimensions in range and azimuth, and $I_{3}$ denotes the number of SAR images. The data stack can be modeled by

$$
\overline{\mathcal{G}}(\mathbf{S}, \mathbf{P})=\mathcal{A} \odot \exp \left\{-j\left(\frac{4 \pi}{\lambda r} \mathbf{S} \otimes \mathbf{b}+\frac{4 \pi}{\lambda} \mathbf{P} \otimes \boldsymbol{\tau}\right)\right\},
$$

where $\overline{\mathcal{G}}$ is the modeled tensor, $\mathcal{A}$ denotes the modeled amplitude tensor, $\mathbf{b} \in \mathbb{R}^{I_{3}}$ is the vector of the spatial baselines, $\boldsymbol{\tau} \in \mathbb{R}^{I_{3}}$ is a warped time variable, e.g. $\boldsymbol{\tau}=\mathbf{t}$ for a linear motion, and $\boldsymbol{\tau}=\sin \left(2 \pi\left(\mathbf{t}-t_{0}\right)\right)$ for a seasonal motion model with temporal baseline $\mathbf{t}$ and time offset $t_{0} . \mathbf{S} \in \mathbb{R}^{I_{1} \times I_{2}}$ and $\mathbf{P} \in \mathbb{R}^{I_{1} \times I_{2}}$ are the unknown elevation and deformation maps to be estimated, respectively, $\lambda$ is the wavelength of the radar signals and $r$ denotes the range between radar and the observed object. The symbol $\odot$ and $\otimes$ denote element-wise and the outer product, respectively.

For our TanDEM-X bistatic InSAR data stack, it can be modeled without the deformation induced phase as:

$$
\overline{\mathcal{G}}(\mathbf{S})=\mathcal{A} \odot \exp \left\{-j\left(\frac{4 \pi}{\lambda r} \mathbf{S} \otimes \mathbf{b}\right)\right\},
$$

where the elevation matrix $\mathbf{S}$ is of the most interest.

\section{ROBUST LOW RANK TENSOR DECOMPOSITION}

Robust low rank tensor decomposition minimizes the rank of the tensor with $L_{0}$ norm of the approximation error

$$
\{\hat{\mathcal{X}}, \hat{\mathcal{E}}\}=\underset{\mathcal{X}, \mathcal{E}}{\operatorname{argmin}} \operatorname{rank}(\mathcal{X})+\gamma\|\mathcal{E}\|_{0}, \quad \text { s.t. } \mathcal{X}+\mathcal{E}=\mathcal{G},
$$

where $\mathcal{G}$ is the observed InSAR data stack, $\mathcal{X}$ is the true InSAR tensor, $\mathcal{E}$ models the tensor of sparse outliers, and $\hat{\mathcal{X}}, \hat{\mathcal{E}}$ are the recovered outlier-free tensor and the estimated outlier
Table 1. Numerical analysis of the results shown in Figure 1

\begin{tabular}{c|c|c|c}
\hline \hline & PSI & SqueeSAR & The proposed method \\
\hline SD $(\mathrm{m})$ & 156.22 & 5.68 & 2.35 \\
\hline bias $(\mathrm{m})$ & -18.62 & -0.83 & -0.18 \\
\hline \hline
\end{tabular}

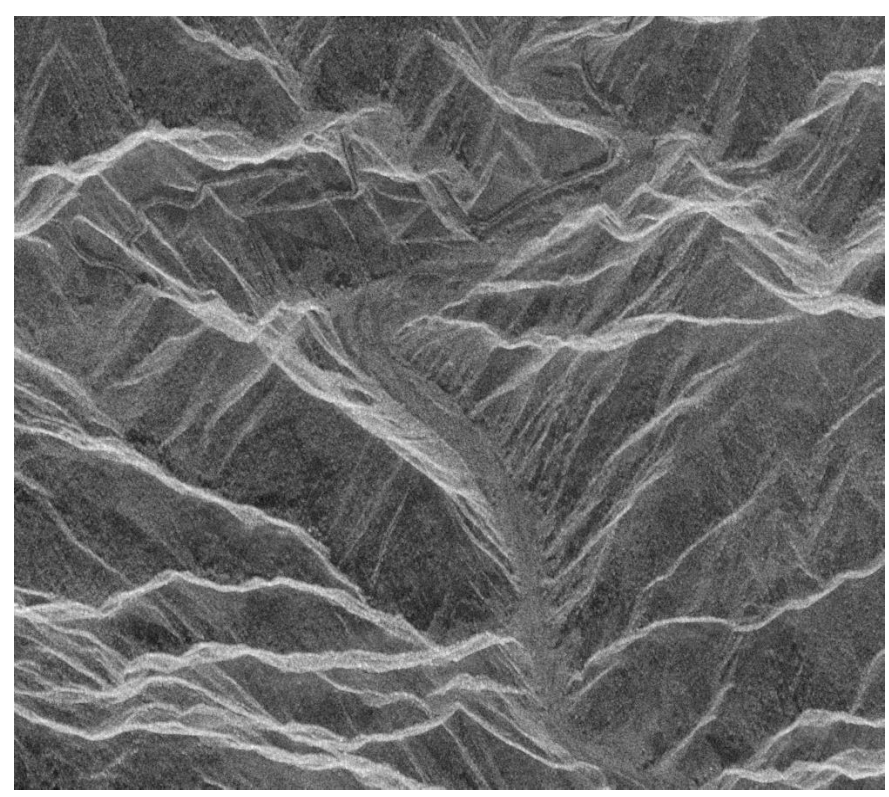

Fig. 3. The study mountain area of TanDEM-X dataset (shown in log-scale).

tensor, respectively. $\operatorname{rank}(\mathcal{X})$ refers to the multilinear rank of $\mathcal{X},\|\mathcal{E}\|_{0}$ denotes the $L_{0}$ norm of $\mathcal{E}$, i.e. $\|\mathcal{E}\|_{0}=\|\operatorname{vec}(\mathcal{E})\|_{0}$, and $\gamma$ is the regularization parameter.

This problem is NP hard, due to the minimization of the multilinear rank and the $L_{0}$ norm. Regarding this, [8] suggested to replace (3) by the following convex optimization problem

$$
\{\hat{\mathcal{X}}, \hat{\mathcal{E}}\}=\underset{\mathcal{X}, \mathcal{E}}{\operatorname{argmin}}\|\mathcal{X}\|_{*}+\gamma\|\mathcal{E}\|_{1}, \quad \text { s.t. } \mathcal{X}+\mathcal{E}=\mathcal{G} .
$$

It relaxes the optimization by replacing the tensor multilinear rank with the tensor nuclear norm $\|\mathcal{X}\|_{*}$ which is the sum of the nuclear norms $\sum_{n}\left\|\mathbf{X}_{(n)}\right\|_{*}$ of the $N$ mode- $n$ unfoldings of $\mathcal{X}$, i.e. $\|\mathcal{X}\|_{*}=\sum_{n}\left\|\mathbf{X}_{(n)}\right\|_{*}$, and by replacing the tensor $L_{0}$ norm with the convex $L_{1}$ norm, i.e. $\|\mathcal{E}\|_{1}=\|\operatorname{vec}(\mathcal{E})\|_{1}$.

This optimization problem can be solved by the framework of Alternating Direction Method of Multipliers (ADMM) [9]. Given the predefined convergence condition, the optimal reconstruction $\hat{\mathcal{X}}$ can be found. To this end, by applying periodogram on $\hat{\mathcal{X}}$, elevations can be robustly retrieved. 

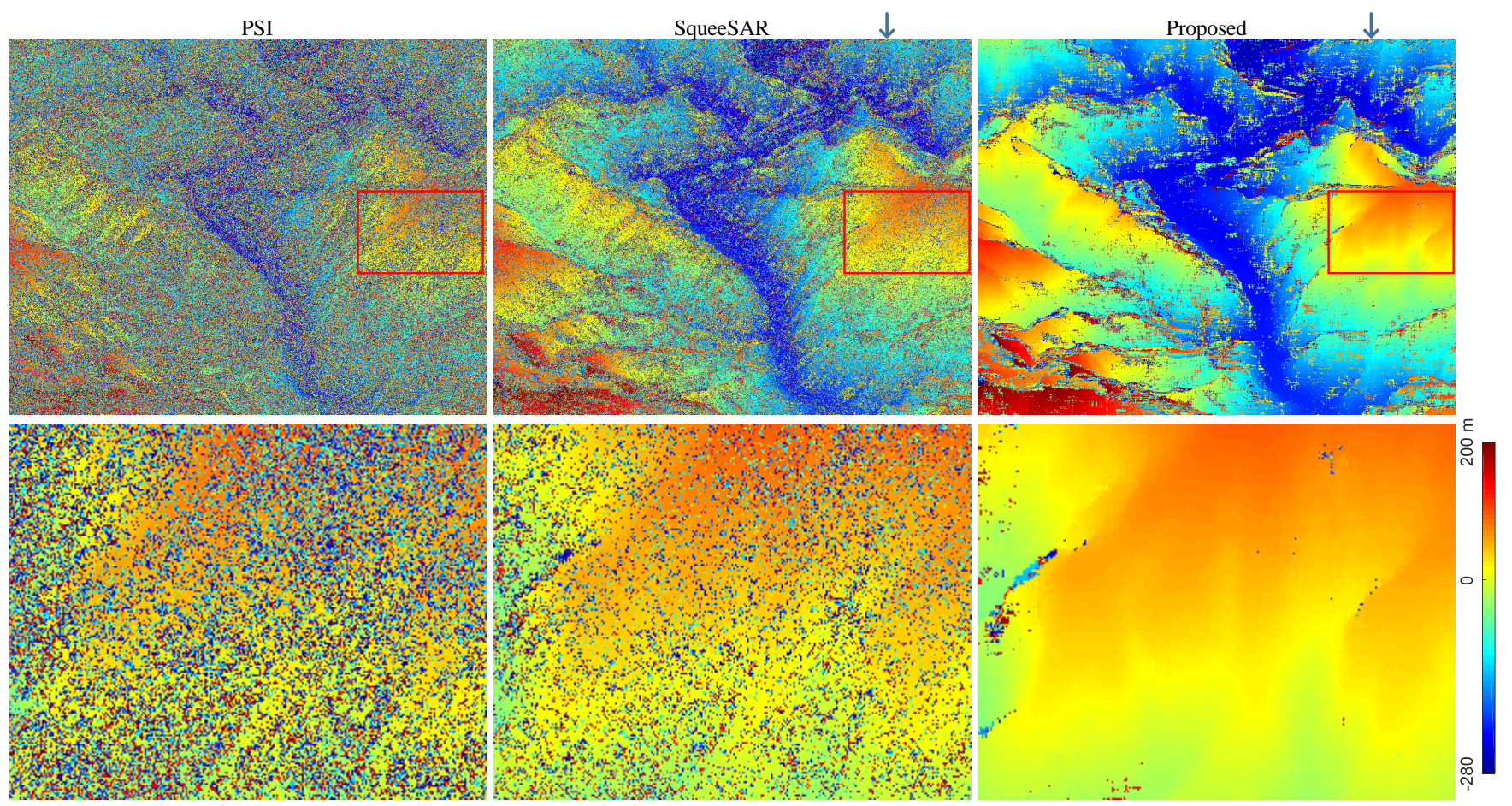

Fig. 4. (First row) The 3D reconstruction results by PSI, SqueeSAR and the proposed method of the TanDEM-X InSAR stack. (Second row) The results of one zoom-in part on the mountain. The proposed method can demonstrate clearer pattern of height variation than SqueeSAR and PSI.

\section{EXPERIMENTS}

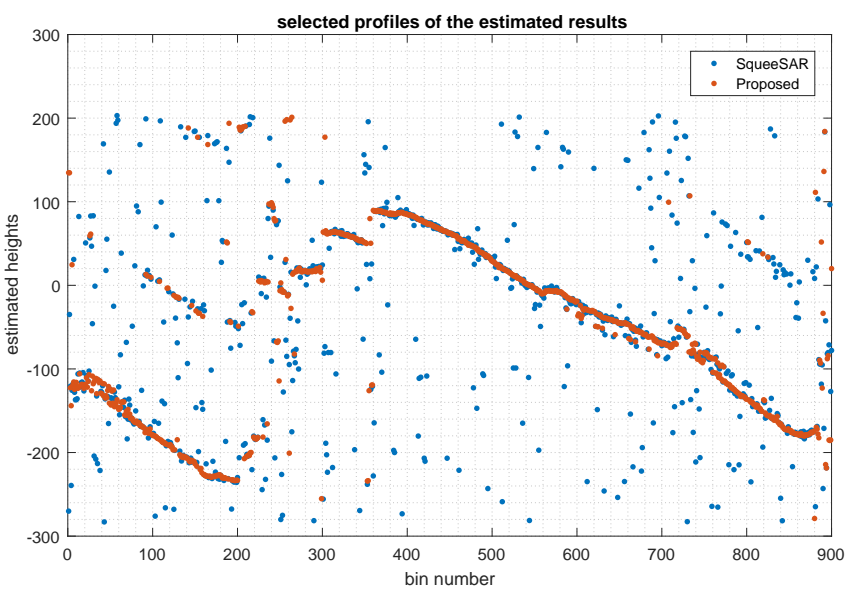

Fig. 5. The selected profiles from the reconstructed results of SqueeSAR and the proposed method, respectively. The profile from the proposed method shows obviously much fewer outliers.

\subsection{Simulations}

We simulated a multi-pass InSAR phase stack of $512 \times 512$ pixels by 7 interferograms with the elevation pattern shown in Figure 1 (top-left). The spatial baseline was chosen to be similar to the typical TanDEM-X baseline range. In order to simulate the mountainous scenario, DS were generated from the ground truth phase stack with the coherence matrix shown in 2. Also, sparse outliers were added to the stack by randomly replacing $20 \%$ of pixels from the stack with uniformly distributed phases. We compared the elevations estimated by PSI, SqueeSAR [5] and the proposed method in Figure 1. Moreover, the standard deviations (SD) and the biases of the estimates are listed in Table1.

As shown in the results, it is obvious to see that PSI cannot correctly estimate elevations for more than $42 \%$ of the pixels. By performing adaptive filtering, SqueeSAR can mitigate most of the wrongly estimated points. This is mainly because of the pixels are simulated according to a perfect DS model which allows an optimal performance of the homogeneous sample selection via statistical test. However, we can still see that noisy points exist in the result from SqueeSAR, which is mainly due to the outliers. In comparison, the proposed method can robustly reconstruct elevation map from 
the DS tensor. As demonstrated by the numerical analysis in Table 1. the proposed method can outperform SqueeSAR by a factor of two in the accuracy of the elevation estimates.

\subsection{Real data}

For testing the performance of the proposed method on real data, we selected one TanDEM-X InSAR stack with 7 images. As illustrated in Figure 3 the study area is shown by the mean amplitude image (log-scale) with the size of $900 \times 1000$ pixels. We can see this complex area mainly composes of mountains and a long valley in-between. For the elevation reconstruction, we utilized PSI, SqueeSAR and the proposed method and compared the results in Figure 4.

The performance of the three algorithm on real data is consistent with the simulation in the previous section. For most parts in the scene, PSI cannot reconstruct reliable elevations from DSs. Although SqueeSAR can retrieve the elevations of most parts in the scene, many points are still incorrectly estimated. Not surprisingly, SqueeSAR does not perform as well as in the simulation, because many pixels do not perfectly follow a DS model. In comparison, the proposed method can robustly recover the height map of nearly the whole scene by efficiently exploit the neighborhood information using low rank tensor decomposition. Improvements are particularly prominent for the pixels on large slopes and on the valley. For a detailed comparison, the estimates of one zoom-in area on the mountain are present in the second row of Figure 4. In addition, Figure 5 shows two height profiles (pointed out by the blue arrow in Figure 4) from the results of SqueeSAR and the proposed method. As shown from the zoom-in parts, the proposed method can demonstrate clearer pattern of height variation than SqueeSAR. Also, in Figure 5 , the profile from the proposed method shows obviously much fewer outliers. However, there are still many pixels which cannot be correctly estimated in the result of the proposed method, especially along the contour lines of mountains. One plausible reason may be that layover happens in those areas.

\section{CONCLUSIONS}

In this paper, we proposed a novel method for elevation reconstruction in complex mountainous areas based on the low rank property inherent in the complex InSAR data stack. According to the simulated experiments under typical settings, the proposed method can achieve reliable estimates even with a limited number of SAR images (less than ten) and outperforms the state-of-the-art methods such as SqueeSAR by a factor of more than two in terms of the accuracy of the height estimates.

According to our experiments, the proposed approach is suitable for operational processing on large areas, as it can be easily parallelized by carrying out patch-wisely to avoid highly computational costs induced by the low rank decomposition of large 3D tensors.

\section{REFERENCES}

[1] Alessandro Ferretti, Claudio Prati, and Fabio Rocca, "Permanent scatterers in SAR interferometry," IEEE Transactions on geoscience and remote sensing, vol. 39, no. 1, pp. 8-20, 2001.

[2] Paola Rizzoli, Michele Martone, Carolina Gonzalez, Christopher Wecklich, Daniela Borla Tridon, Benjamin Bräutigam, Markus Bachmann, Daniel Schulze, Thomas Fritz, Martin Huber, et al., "Generation and performance assessment of the global tandem-x digital elevation model," ISPRS Journal of Photogrammetry and Remote Sensing, vol. 132, pp. 119-139, 2017.

[3] J. Kang, Y. Wang, M. Krner, and X. X. Zhu, "Robust Object-Based Multipass InSAR Deformation Reconstruction," IEEE Transactions on Geoscience and Remote Sensing, vol. 55, no. 8, pp. 4239-4251, Aug 2017.

[4] Yuanyuan Wang, Xiao Xiang Zhu, and Richard Bamler, "Retrieval of phase history parameters from distributed scatterers in urban areas using very high resolution SAR data," ISPRS Journal of Photogrammetry and Remote Sensing, vol. 73, pp. 89 - 99, 2012.

[5] Alessandro Ferretti, Alfio Fumagalli, Fabrizio Novali, Claudio Prati, Fabio Rocca, and Alessio Rucci, "A new algorithm for processing interferometric data-stacks: SqueeSAR," IEEE Transactions on Geoscience and Remote Sensing, vol. 49, no. 9, pp. 3460-3470, 2011.

[6] Jian Kang, Yuanyuan Wang, Marco Körner, and Xiao Xiang Zhu, "Object-based insar deformation reconstruction with application to bridge monitoring," in Geoscience and Remote Sensing Symposium (IGARSS), 2016 IEEE International. IEEE, 2016, pp. 6871-6874.

[7] Jian Kang, Yuanyuan Wang, Michael Schmitt, and Xiao Xiang Zhu, "Object-based Multipass InSAR via Robust Low Rank Tensor Decomposition," IEEE Transactions on Geoscience and Remote Sensing, in press,2018.

[8] Donald Goldfarb and Zhiwei Qin, "Robust low-rank tensor recovery: Models and algorithms," SIAM Journal on Matrix Analysis and Applications, vol. 35, no. 1, pp. 225253, 2014.

[9] Stephen Boyd, Neal Parikh, Eric Chu, Borja Peleato, and Jonathan Eckstein, "Distributed optimization and statistical learning via the alternating direction method of multipliers," Foundations and Trends $\mathrm{R}$ in Machine Learning, vol. 3, no. 1, pp. 1-122, 2011. 